\title{
Multilevel Models of Krypton for the Application to Stepwise Ionization Relaxation Processes
}

\author{
L. Bergmann and F. Demmig \\ Institut für Plasmaphysik der Technischen Universität Hannover \\ (Z. Naturforsch. 32a, 1245-1248 [1977]; received September 3, 1977)
}

\begin{abstract}
A four step model and two five step models of krypton are developed for application to stepwise ionization relaxation. The four step model is preferred out of practical and economical grounds. The corresponding rate equations for collisional excitation and ionization are given assuming ladder climbing for the electrons. Selected results of an application of the model to ionization relaxation in shock waves are reported.
\end{abstract}

\section{Introduction}

Recent investigations of ionization relaxation ${ }^{1-6}$ have shown that multistep models of ionization are superior to the two step models* allowing only for one intermediate step of excitation between the ground level and the ionization level.

They are indispensible when measurements of population densities of higher excited levels shall be utilized for comparison of theory and experiment. The reaction equations for stepwise collisional excitation of an atom A read,

$$
A_{0}+A_{i} \stackrel{k_{i j}^{\mathrm{a}}}{\longrightarrow} A_{0}+A_{j}, \quad A_{i}+e \stackrel{k_{i j}^{\mathrm{e}}}{\longrightarrow} A_{j}+e,
$$

where the subscript denotes the level of excitation and $k_{i j}$ is the rate coefficient for the transition from level $i$ to level $j$. The letters a and e indicate atomatom collisions and electron-atom collisions, respectively.

In devising a practical multilevel model one has to reduce the actual level scheme of the atom in question to a small number of energy levels in order to obtain a manageable set of rate equations for the stepwise excitation/de-excitation. This procedure will be described for krypton in Section 2 . For the resulting quasilevels the rate equations are formulated in Section 3. Section 4 gives a discussion of results obtained by applying the proposed krypton models to ionization relaxation.

\section{Model Atom for Multistep Processes}

For $q$ step models of ionization relaxation, the actual atomic energy levels are combined to form

* To name only some authors dealing with the two step model: Ref. 7 through 15.

Reprint requests to Dr. Frank Demmig, Institut für Plasmaphysik, Callinstraße 38, D-3000 Hannover 1. $q-1$ groups of closely spaced energy levels ${ }^{2,4}$. Each group is represented by a quasilevel with an energy value defined by the weighted mean

$$
\varepsilon=\frac{1}{\gamma} \sum_{l=1}^{N} \gamma_{l} \varepsilon_{l},
$$

where $\gamma_{l}, \quad(l=1,2, \ldots, N)$, are the statistical weights of the corresponding $N$ actual levels with energies $\varepsilon_{1}, \varepsilon_{2}, \ldots, \varepsilon_{N}$. The statistical weight $\gamma$ of the quasilevel is defined by

$$
\gamma=\sum_{l=1}^{N} \gamma_{l}
$$

The partition function then can be calculated for all quasi levels in the usual way since the partial sum corresponding to the particular group of actual levels may be written

$Q_{\text {partial }}=\sum_{l=1}^{N} \gamma_{l} \exp \left(-\varepsilon_{l} / k T\right)=\gamma \exp (-\varepsilon / k T)$

with a relative error of about

$$
0.5 \times(\varepsilon / k T)^{2} \times \sum_{l=1}^{N} \gamma_{l}\left(1-\varepsilon_{l} / \varepsilon\right)^{2} / \gamma,
$$

which is small for levels $\varepsilon_{l}$ spread not too widely. Defining the population density $n_{i}$ of a quasilevel i by the sum of the $N_{i}$ combined individual population densities

$$
n_{i}=\sum_{l=1}^{N_{i}} n_{l}^{(i)},
$$

in thermodynamic equilibrium the population densities $n_{i}{ }^{*}$ of the quasilevels then are related to the number density $n$ of neutrals by

$$
\frac{n_{i}^{*}}{n}=\frac{\gamma_{i}}{Q} \exp \left(-\frac{\varepsilon_{i}}{k T}\right) .
$$


Table 1. Four step model (A) and five step models (B, C) for krypton with energies $\varepsilon$, statistical weights $\gamma$, and effective quantum numbers $\eta$ of quasilevels.

\begin{tabular}{lrrrrrrrrr}
\hline Model $\mathrm{A}$ & \multicolumn{1}{c}{$\mathrm{B}$} & \multicolumn{7}{c}{$\mathrm{C}$} \\
\hline $\begin{array}{l}\text { quasi- } \\
\text { level }\end{array}$ & & $\gamma$ & $\eta$ & $\varepsilon$ & $\gamma$ & $\eta$ & $\varepsilon$ & $\gamma$ & $\eta$ \\
\hline 0 & 0 & 1 & & 0 & 1 & & 0 & 1 & \\
1 & 10.18 & 12 & 3 & 10.18 & 12 & 3 & 9.96 & 8 & 2.5 \\
2 & 11.99 & 84 & 5 & 11.47 & 24 & 4.5 & 10.62 & 4 & 3.5 \\
3 & 13.40 & 400 & 8 & 12.20 & 60 & 5.5 & 11.99 & 84 & 5 \\
4 & & & & 13.40 & 400 & 8 & 13.40 & 400 & 8 \\
+ & 14.00 & & & 14.00 & & & 14.00 & & \\
\hline
\end{tabular}

This equation holds by consequence of Eq. (4), $Q=\sum_{i} \gamma_{i} \exp \left(-\varepsilon_{i} / k T\right)$ being the total atomic partition function. Hence the backward reaction rate coefficient $k_{j i}$ for a collisional de-excitation from quasilevel $j$ to $i$ can be determined via the principle of detailed balancing,

$$
k_{j i}=\frac{n_{i}^{*}}{n_{j}^{*}} k_{i j}=\frac{\gamma_{i}}{\gamma_{j}} k_{i j} \exp \left(\frac{\varepsilon_{i j}}{k T}\right),
$$

where $\varepsilon_{i j}=\varepsilon_{j}-\varepsilon_{i}$.

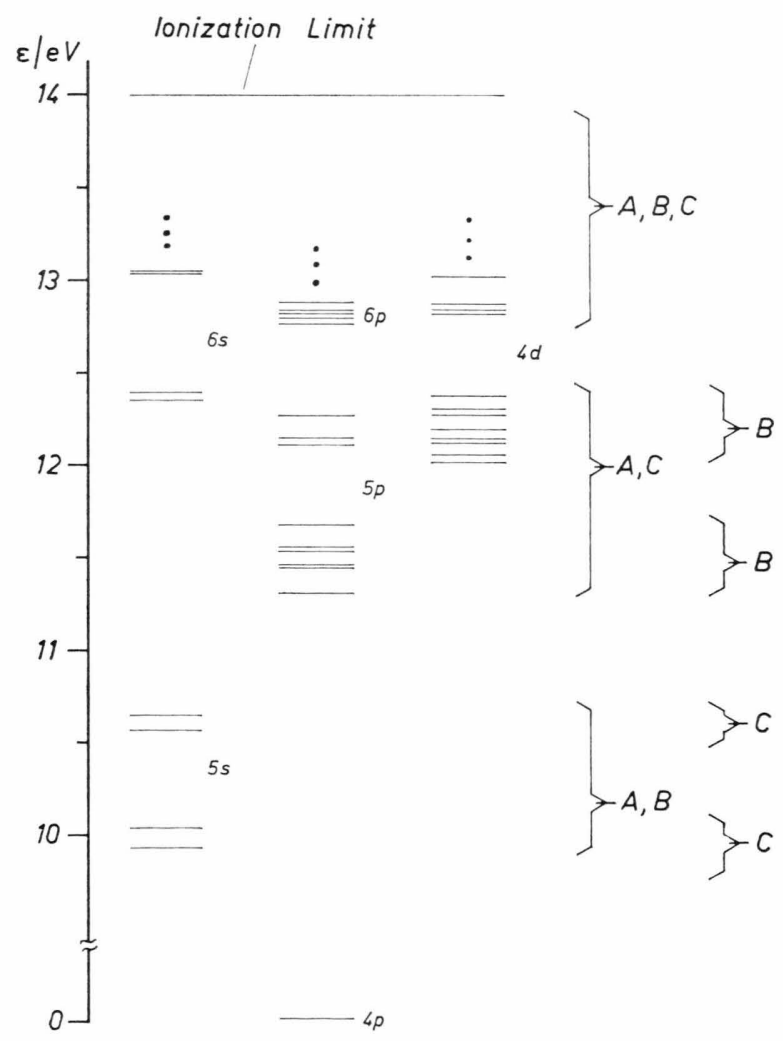

Fig. 1. Level scheme of krypton and quasilevels of the models $\mathrm{A}, \mathrm{B}$, and $\mathrm{C}$.
Our aim was the development of a refined model based on stepwise ionization which on the one hand is as simple as possible - i.e. consisting of few steps - and which on the other hand corresponds well to the actual level structure of krypton. Table 1 shows a four step model and two five step models. The energy values and the statistical weights have been determined by formulae (2) and (3).

The highest quasilevel comprises all high lying levels of krypton, its statistical weight is an educated guess being discussed in more detail in Section 4 . The effective quantum numbers refer to the procedure of Hollenbach and Salpeter described in Section 3 in the choice of which we were guided by the argon models of Hollenbach and Salpeter ${ }^{2}$, Nishimura et al. ${ }^{3}$, and Wojciechowski and Weymann ${ }^{4}$. The configuration of atomic levels represented by a quasilevel is indicated in the level scheme of krypton in Fig. 1 by vertical brackets.

\section{Rate Equations for Ionization Relaxation}

Since we apply the multistep models to the ionization relaxation in shock tube plasmas with shock Mach numbers about 10 , radiation processes can be neglected ${ }^{3,16}$. We assume ladder climbing, i.e. electronic transition only to neighbouring quasilevels, $j_{\searrow j-1}^{j+1}$, but allow also for direct ionization, which however proves to give only a small contribution. The rate equations then read in the case of the four step model

$$
\begin{aligned}
\mathrm{d} n_{1} / \mathrm{d} t= & n_{0}^{2} k_{01}^{\mathrm{a}}+n_{0} n_{2} k_{21}^{\mathrm{a}}-n_{0} n_{1}\left(k_{10}^{\mathrm{a}}+k_{12}^{\mathrm{a}}+k_{1+}^{\mathrm{a}}\right) \\
& +n_{0} n_{e}^{2} k_{+1}^{\mathrm{a}}+n_{0} n_{e} k_{01}^{\mathrm{e}}+n_{e} n_{2} k_{21}^{\mathrm{e}} \\
& -n_{e} n_{1}\left(k_{10}^{\mathrm{e}}+k_{12}^{\mathrm{e}}+k_{1+}^{\mathrm{e}}\right)+n_{e}^{3} k_{+1}^{\mathrm{e}}
\end{aligned}
$$

$\mathrm{d} n_{2} / \mathrm{d} t=n_{0} n_{1} k_{12}^{\mathrm{a}}+n_{0} n_{3} k_{32}^{\mathrm{a}}-n_{0} n_{2}\left(k_{21}^{\mathrm{a}}+k_{23}^{\mathrm{a}}+k_{2+}^{\mathrm{a}}\right)$

$+n_{0} n_{e}^{2} k_{+2}^{\mathrm{a}}+n_{e} n_{1} k_{12}^{\mathrm{e}}+n_{e} n_{3} k_{32}^{\mathrm{e}}$

$-n_{e} n_{2}\left(k_{21}^{\mathrm{e}}+k_{23}^{\mathrm{e}}+k_{2+}^{\mathrm{e}}\right)+n_{e}^{3} k_{+2}^{\mathrm{e}}$,

$\mathrm{d} n_{3} / \mathrm{d} t=n_{0} n_{2} k_{23}^{\mathrm{a}}-n_{0} n_{3}\left(k_{32}^{\mathrm{a}}+k_{3+}^{\mathrm{a}}\right)+n_{0} n_{e}^{2} k_{+3}^{\mathrm{a}}$

$+n_{e} n_{2} k_{23}^{\mathrm{e}}-n_{e} n_{3}\left(k_{32}^{\mathrm{e}}+k_{3+}^{\mathrm{e}}\right)+n_{e}^{3} k_{+3}^{\mathrm{e}}$,

$$
\begin{aligned}
\mathrm{d} n_{e} / \mathrm{d} t= & n_{0} n_{1} k_{1+}^{\mathrm{a}}+n_{0} n_{2} k_{2+}^{\mathrm{a}}+n_{0} n_{3} k_{3+}^{\mathrm{a}} \\
& -n_{0} n_{e}^{2}\left(k_{+1}^{\mathrm{a}}+k_{+2}^{\mathrm{a}}+k_{+3}^{\mathrm{a}}\right) \\
& +n_{e} n_{1} k_{1+}^{\mathrm{e}}+n_{e} n_{2} k_{2+}^{\mathrm{e}}+n_{e} n_{3} k_{3+}^{\mathrm{e}} \\
& -n_{\mathrm{e}}^{3}\left(k_{+1}^{\mathrm{e}}+k_{+2}^{\mathrm{e}}+k_{+3}^{\mathrm{e}}\right) .
\end{aligned}
$$


In the case of model B or C a fourth step has to be added

$$
\begin{aligned}
\mathrm{d} n_{4} / \mathrm{d} t= & n_{0} n_{3} k_{34}^{\mathrm{a}}-n_{0} n_{4}\left(k_{43}^{\mathrm{a}}+k_{4+}^{\mathrm{a}}\right)+n_{0} n_{e}^{2} k_{+4}^{\mathrm{a}} \\
& +n_{e} n_{3} k_{34}^{\mathrm{e}}-n_{e} n_{4}\left(k_{43}^{\mathrm{e}}+k_{4+}^{\mathrm{e}}\right)+n_{\mathrm{e}}^{3} k_{+4}^{\mathrm{e}},
\end{aligned}
$$

and in Eqs. (10) and (11) additional terms,

$-n_{0} n_{3} k_{34}^{\mathrm{a}}+n_{0} n_{4} k_{43}^{\mathrm{a}}-n_{e} n_{3} k_{34}^{\mathrm{e}}+n_{e} n_{4} k_{43}^{\mathrm{e}}$ and

$+n_{0} n_{4} k_{4+}^{\mathrm{a}}-n_{0} n_{e}^{2} k_{+4}^{\mathrm{a}}+n_{e} n_{4} k_{4+}^{\mathrm{e}}-n_{e}^{3} k_{+4}^{\mathrm{e}}$

have to be considered, respectively.

The rate coefficients $k_{i j}^{\mathrm{a}}$ for atom-atom collisions and $k_{i j}^{\mathrm{e}}$ for electron-atom collisions are given by

$k_{i j}=4 S_{i j}(2 \pi \mu)^{-1 / 2}(k T)^{3 / 2}\left(\frac{\varepsilon_{i j}}{k T}+2\right) \exp \left(-\frac{\varepsilon_{i j}}{k T}\right)$,

where $\mu$ denotes the reduced mass and $S_{i j}$ are the cross section constants. This expression for the rate coefficients is valid in the case of a constant slope of the corresponding cross section near the threshold and a Maxwell distribution of the electrons. For the calculation of the rate coefficients $k_{i j}^{\mathrm{e}}$ one has to use the electron temperature $T_{e}$, which is different from the temperature $T_{h}$ of heavy particles throughout the relaxation zone. $k_{i+}$ is the rate coefficient for direct ionization from the $i$ th quasilevel, which is formed in analogy to Equation (13). For the backward rate coefficients $k_{j i}$ and $k_{+4}$ see Equation (7).

The number of cross section constants $S_{i j}^{\mathrm{a}}$ can be reduced by the semiempirical procedure of Hollenbach and Salpeter ${ }^{2}$, which relates the $S_{i j}^{\mathrm{a}}$ to effective quantum numbers,

$$
\begin{aligned}
& S_{i j}^{\mathrm{a}}=S_{12}^{\mathrm{a}}\left(\frac{\eta_{2}^{-2}+12^{-2}}{\eta_{j}^{-2}+12^{-2}}\right)^{2}, \\
& S_{i+}^{\mathrm{a}}=S_{1+}^{\mathrm{a}}\left(\frac{\eta_{1}^{-2}+12^{-2}}{\eta_{i}{ }^{-2}+12^{-2}}\right)^{2} .
\end{aligned}
$$

The first step cannot be treated by Eq. (14) because of its relatively large energy difference, hence there are now three parameters left: $S_{01}^{\mathrm{a}}, S_{12}^{\mathrm{a}}$, and $S_{1+}^{\mathrm{a}}$. Since Hollenbach and Salpeter do not give a precise definition of the effective quantum numbers, the reduction of parameters is not as complete as it appears to be ${ }^{5}$.

\section{Discussion}

The krypton models A, B, and C have been used ${ }^{5}$ for the description of ionization relaxation in shock waves with Mach numbers in the range of 9 through 12. Because these shock waves were generated in a shock tube, the effect of boundary layers and of the weakly unsteady flow had to be taken into account ${ }^{6}$. The calculated values of the electron density $n_{e}$, the population densities $n_{1}$ and $n_{2}$, and the density $n_{0}$ of particles in the ground level have been compared with preliminary experimental values resulting from interferometric, absorption, and emission experiments $16,17,18$. The following set of cross section constants gives good agreement of theoretical and experimental values in the case of the four step model,

$S_{01}^{\mathrm{a}}=2 \times 10^{-5}, S_{12}^{\mathrm{a}}=7 \times 10^{-2}, \quad S_{1+}^{\mathrm{a}}=7.3 \times 10^{-3}$, $S_{01}^{\mathrm{e}}=6.6 \times 10^{-3}, \quad S_{12}^{\mathrm{e}}=1, \quad S_{23}^{\mathrm{e}}=2=S_{3+}^{\mathrm{e}}\left[\mathrm{m}^{2} / \mathrm{Ws}\right]$.

For error bounds and further information about the fluid dynamical aspect we refer to Reference ${ }^{6}$.

Since the energy of the highest quasilevel and its statistical weight cannot be determined precisely, we varied these parameters to study their influence. The variation of the energy value $\varepsilon_{3}$ by $0.2 \mathrm{eV}$ resulted in variations of the population densities: $n_{1}$ by $2 \%, n_{2}$ by $12 \%, n_{3}$ by $17 \%$, approximately. The variation of the statistical weight $\gamma_{3}$ within a wide range of 400 through 2000 practically did not influence the population densities $n_{1}$ and $n_{3}$ whereas $n_{2}$ decreased nearly linearly by $40 \%$ with increasing $\gamma_{3}$. Since the experimental error of the population densities presently cannot be reduced to less than $30 \%$, this uncertainty both of the energy and the statistical weight of the highest quasilevel is not crucial.

In our application of the krypton models to ionization relaxation we concentrated on the four step model A for two reasons. First, a practical model should consist of as few steps as possible to avoid an unnecessarily high number of cross section constants. Second, every additional step enlarges the functional matrix of the system of rate equations and thus largely influences the computation time. Since the rate equations in the unsteady case have to be solved not only along one particle path but throughout the flow field 6 , the computation time in the case of the four step model is about 70 seconds on a CDC CYBER 76 machine, which time could hardly be substantially exceeded for evaluation of extensive series of measurements. Moreover, test computations showed that the five step models B and $\mathrm{C}$ offer a differentiation which could not be turned into profit in view of the present accuracy of measurement. 


\section{Acknowledgement}

We should like to thank W. Bötticher and the members of the shock tube group of our institute for valuable discussions.

1 P. C. T. de Boer and P. R. Grimwood, Bull. Amer. Phys. Soc. 13, 808 [1968].

2 D. J. Hollenbach and E. E. Salpeter, J. Chem. Phys. 50, 4157 [1969].

3 M. Nishimura, K. Teshima, and G. Kamimoto, Recent developments in shock tube research, 9th Shock Tube Symposium, Stanford 1973, p. 294.

4 P. H. Wojeiechowski and H. D. Weymann, J. Chem. Phys. 61, 1369 [1974].

5 L. Bergmann, Diplomarbeit, Technische Universität Hannover 1977.

6 F. Demmig, 11th International Symposium on Shock Tubes and Waves. Seattle, July 1977.

7 H. Petschek and S. Byron, Ann. Phys. 1, 270 [1957].

8 J. W. Bond, Phys. Rev. 105, 1683 [1957].

9 K. E. Harwell and R. G. Jahn, Phys. Fluids 7, 214 [1964].
10 A. J. Kelly, J. Chem. Phys. 45, 1723 [1966].

11 P. E. Oettinger and D. Bershader, AIAA J. 5, 1625 [1967].

12 M. I. Hoffert and H. Lien, Phys. Fluids 10, 1769 [1967].

13 H. F. Nelson, Phys. Fluids 16, 2132 [1973].

14 Y. Enomoto, J. Phys. Soc. Japan 35, 1228 [1973] [Erratum Vol. 36, 922].

15 M. S. Oggiano and M. Onorato, Recent Developments in Shock Tube Research, 9th Shock Tube Symposium, Stanford 1973, p. 761.

16 Th. Zaengel, Dissertation, Technische Universität Hannover 1977.

17 W. Schöpper, Diplomarbeit, Technische Universität Hannover 1976.

18 W. Ernst, personal communication. 